\title{
The Italian Tamoxifen Prevention Trial
}

\author{
B. Bonanni and U. Veronesi on behalf of the \\ Italian Tamoxifen Prevention Study
}

Background: On the basis of the $40 \%$ reduction of contralateral breast cancer observed in the most recent metanalysis of adjuvant treatment trials, three large, randomized, placebocontrolled studies were started to evaluate the chemoprevention effect of tamoxifen against breast cancer. In the UK trial no difference in terms of breast cancer occurrence was shown between the tamoxifen and the placebo arm. Conversely, the USA trial showed an almost 50\% reduction of incidence of invasive breast cancer in the tamoxifen group. While these two studies involve healthy women at higher risk, the ongoing Italian trial randomized only well women previously hysterectomized (on the basis that tamoxifen could produce a risk of endometrial cancer), thus selecting in fact a cohort of subjects at low-to-normal risk of breast cancer.

Methods: In 1992, a double-blind placebocontrolled, randomized trial with tamoxifen was started in Italy in healthy women aged 35 to 70 who had had a total hysterectomy for reasons other than cancer. 1412 Women $(26.3 \%)$ had conservation of the ovaries, 2595 (48.3\%) had a bilateral oophorectomy and 998 (18.6\%) had a unilateral oophorectomy. In total, 976 women (18.2\%) had at least one first degree relative or an aunt with breast cancer, $132(2.5 \%)$ had two relatives, and $16(0.3 \%)$ had three relatives with breast cancer. Severe concurrent illness, history of heart disease, endometriosis and previous deep-vein thrombosis were the main exclusion criteria.
Women were randomized to receive tamoxifen $20 \mathrm{mg}$ per day or placebo for 5 years and then followed for another 5 years. While recruitment ended in July 1997, the study is proceeding as planned. Twenty per cent of the participants are also on hormone replacement therapy (HRT). The primary endpoint is the occurrence of breast cancer. The preliminary interim analysis was published in July 1998.

Findings: A total of 5408 subjects were randomized. The median follow-up is 46 months. 41 cases of breast cancer occurred so far, with no deaths from breast cancer.

Overall, there is to date no difference in breastcancer frequency between the placebo (22 cases) and tamoxifen (19) arms. There is instead a statistically significant reduction of breast cancer among women receiving tamoxifen who also used HRT during the trial: eight cases of breast cancer were found among 390 women on HRT taking placebo, compared with one case among 362 women taking tamoxifen (hazard ratio 0.13, 95\% CI, 0.02-1.02).

Compared with the placebo group, there was a significantly increased risk of vascular events and hypertriglyceridaemia among women on tamoxifen. 64 Thromboembolic events occurred in 56 women: 18 women on placebo and 38 on tamoxifen $(\mathrm{p}=0.0053)$. 42 Events were superficial phebitis, while 9 women had a deep-vein thrombosis (6 on tamoxifen, 3 on placebo). There were 14 cerebrovascular ischaemic events ( 5 on placebo, 9 on tamoxifen; $p=0.27$ ). 17 Women had hypertriglyceridaemia ( 2 on placebo, 15 on tamoxifen; $p=0.0013$ ).

Discussion: Our findings appear at variance with those from the NSABP-P1 trial in the USA.

In our cohort of women at low-to-normal risk of breast cancer, the expected protective effects 
of tamoxifen are not yet apparent, with the exception of women using HRT who benefited from the drug administration. These preliminary findings must be confirmed through further investigation.

Notably, no deaths from breast cancer have yet been observed.

A much longer follow-up is certainly needed to quantify the long-term risks and benefits of tamoxifen therapy, especially in view of a future metanalysis of all chemoprevention trials. 


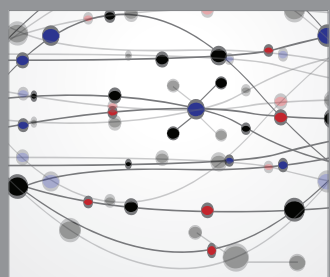

The Scientific World Journal
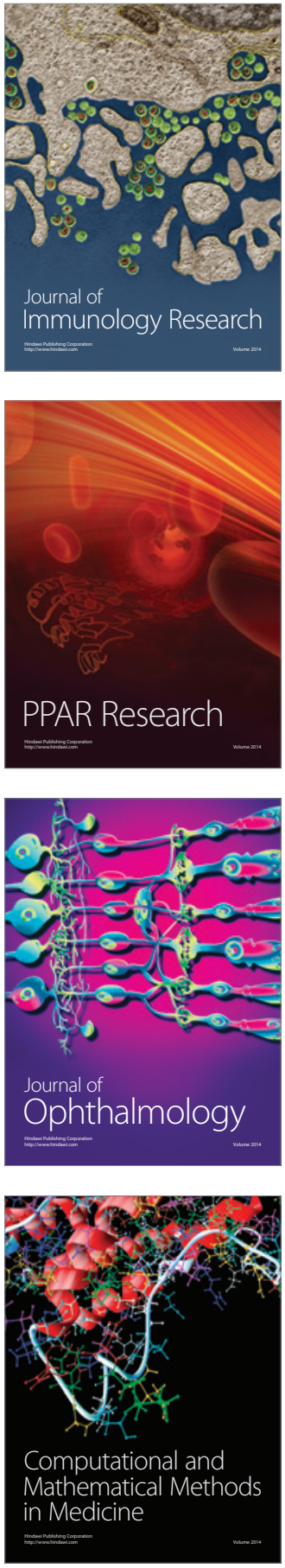

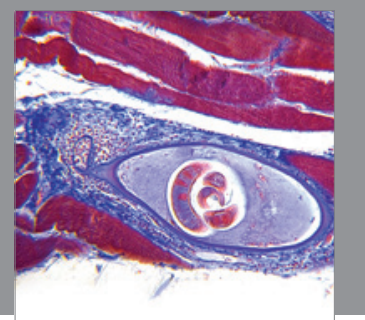

Gastroenterology

Research and Practice
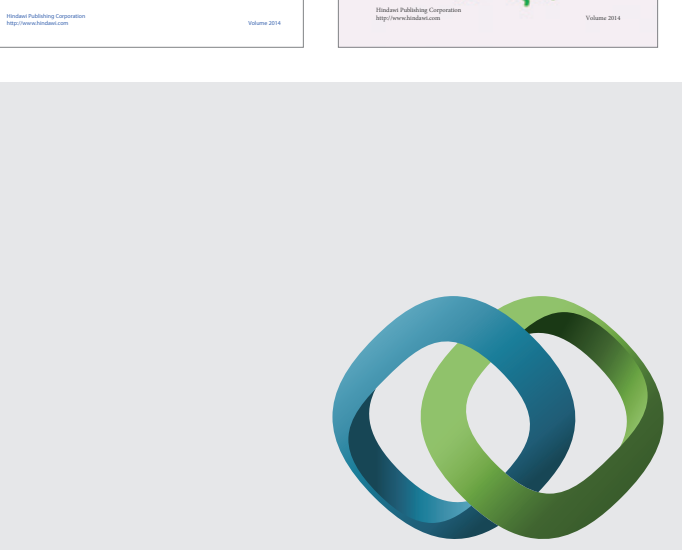

\section{Hindawi}

Submit your manuscripts at

http://www.hindawi.com
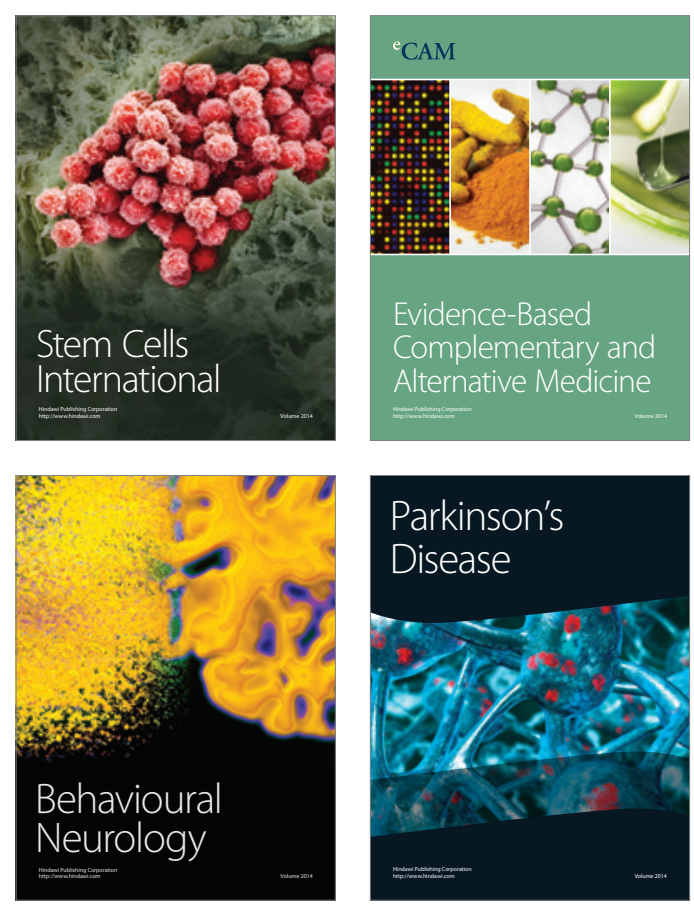

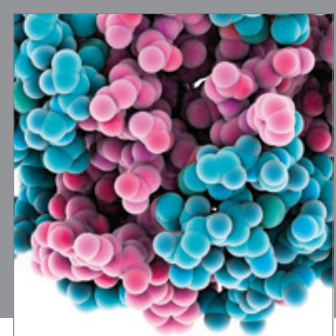

Journal of
Diabetes Research

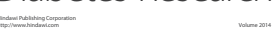

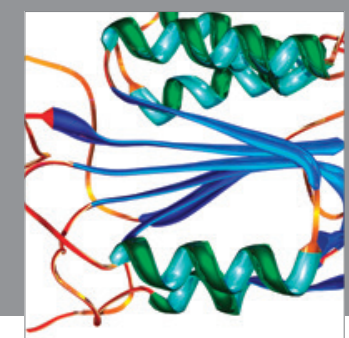

Disease Markers
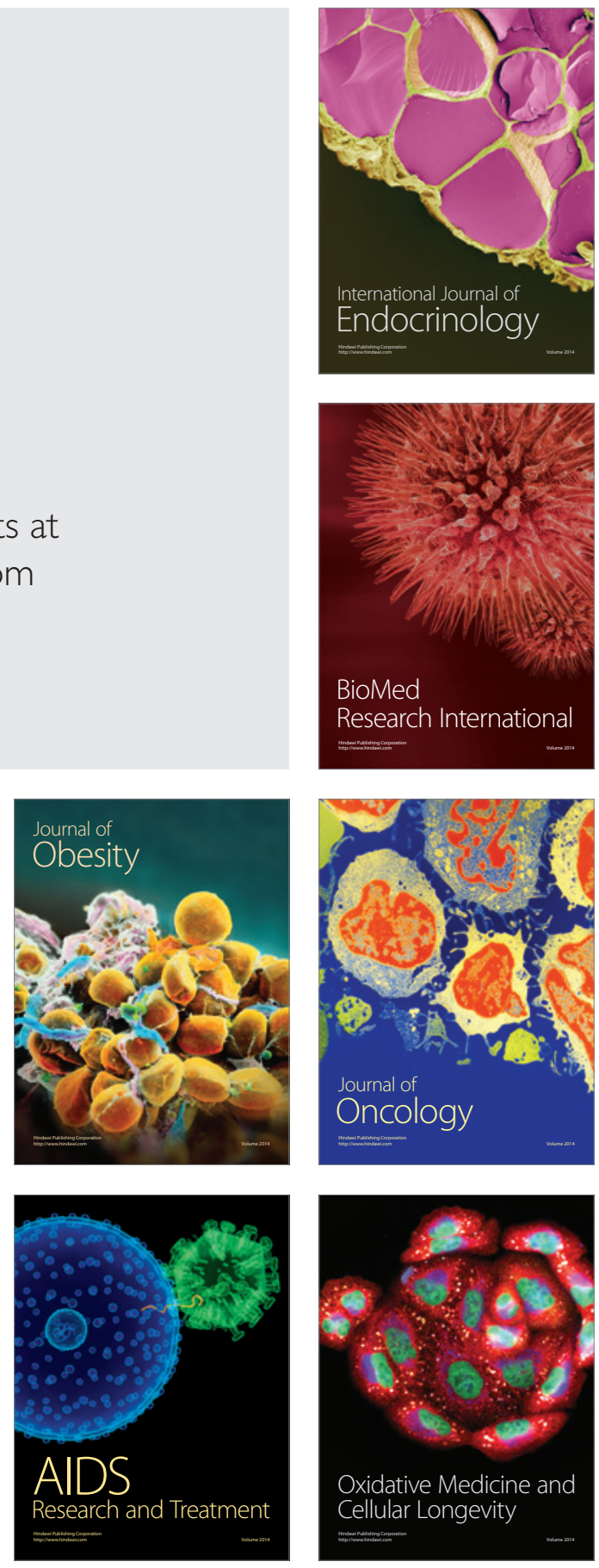